\title{
Consideration of paddock shape and access in subdivision fencing
}

\author{
D. SCOTT \\ Lake Tekapo \\ scott_hc@xtra.co.nz
}

\begin{abstract}
In the potential paddock subdivision of a large property, a simulation study shows a rapidly increasing total fencing requirement as paddock shapes depart from square to rectangular, with it being $6 \%$ greater if length to width ratio is $2: 1$ and $25 \%$ when $4: 1$. It is similar or slightly less for a hexagonal shape with some advantages from machinery or animal behaviour considerations. Fencing a triangular shape would be $21 \%$ greater. Provision of access lanes to all paddocks simplifies management. While a series of ring lanes plus a few connecting lanes in square paddock design increases total fencing by $30 \%$ as compared to $25 \%$ for a central lane plus laterals lanes, it does result in a $9 \%$ reduction in the mean distance (time) needed to move stock between paddocks.
\end{abstract}

Keywords: fencing; subdivision

\section{Introduction}

Improvements in grazing agriculture are through gaining control of animals and pastures, generally through subdivision. The associated fencing is one of the main farm capital expenditures and any efficiency in their design and layout would be desirable.

The main determinants of paddock size and shape are going to be topographic, landscape or soil units, and animal behaviour considerations. Size will generally be determined by the feed needed for a mob for about a week. Shape will generally be determined by landscape or soil type, access to water as well as any direct limitation imposed by topography, with the desirability that a particular paddock includes only one landscape or soil type category. Animal behaviour considerations relate to movement, such as sheep tending to drift along contours rather than vertically, the tendency for sharp fence corners to act as an exclosure, and for sheep tending to camp in the highest point in a paddock or alongside those in adjacent paddocks.

Associated with subdivision is access. There is a tendency for successive subdivisions to grow in an uncoordinated way, with later subdivisions unduly influenced by earlier subdivisions, rather than some final overall plan, and often eventually having to follow an irregular route and open and close 'a hundred gates' to get between different locations. This raises the consideration of providing lanes or access ways between different paddocks for ease of management and stock movement.
Besides all the biological determinants of paddock sizes and shapes mentioned, there are possibly other considerations related to the efficiencies of the general geometry of the fencing layout that can be used as guidelines in successive subdivisions of large areas. The following was a paper exercise looking at the effects of different geometric layouts, assuming a large previously unfenced area within a uniform environment. The geometric considerations are independent of scale, though the example used was thinking of a large property to be ultimately subdivided into 4 ha paddocks. Undoubtedly the considerations were influenced by a background in extensive sheep range-land, with limited existing subdivision, but with a move towards subdivision for special purpose pastures, and the hope that the process could continue to eventual total subdivision.

\section{Paddock Shape}

The first consideration of effect of paddock shape on fencing cost efficiency, is for a single paddock in isolation, considering the area enclosed relative to perimeter fencing needed, and for alternative shapes relative to a square paddock (Fig 1).

The comparison of rectangular shapes with square shaped paddocks indicates the rapidly increasing fencing requirements as the length to width ratio of paddocks increase.

Figure 1 Effect of paddock shape on fencing costs.
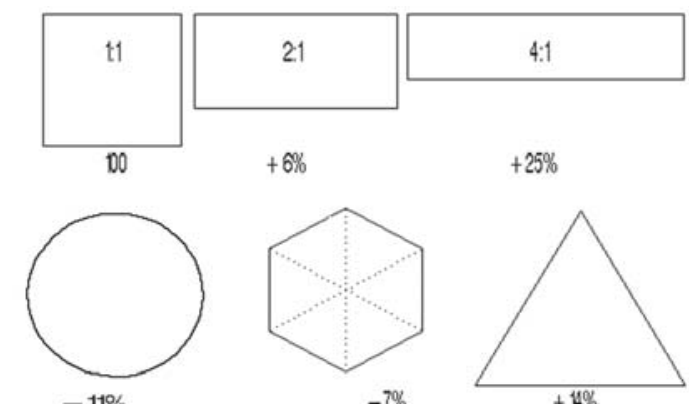

$+6 \%$

$+25 \%$

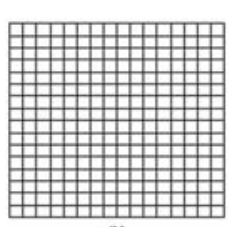

10

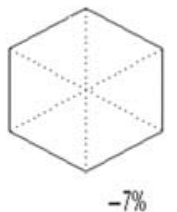

$-7 \%$

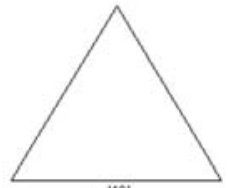

$+14 \%$

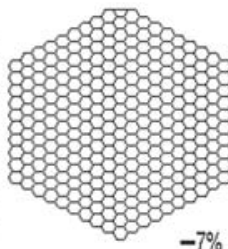

$-7 \%$

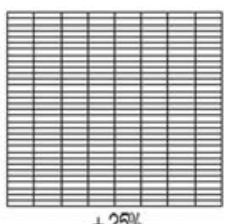


A circular shaped paddock would be the most efficient in terms of the ratio of area enclosed relative to perimeter length, requiring $11 \%$ less fencing than for a square paddocks, and would suit such activities as central pivots irrigators, or lack of blind-spots for animals grazing or machinery. However, it would seldom be practical in the sense that all posts would have to be strainers, and that it would not be possible to juxtapose similar shaped paddocks.

The hexagonal would be a working compromise, with $7 \%$ less fencing than for a square paddock, retaining open obtuse corners for animals grazing or machinery considerations, and easy juxtaposition with adjacent paddocks of the same design.

The relative fencing efficiencies remain the same when extended to continued subdivision using common fences and assuming sharing of property boundary fencing costs. The calculations used a property of 256 paddocks (a) 4 ha $=1024$ ha as that was a simple common denominator for several of the designs. The effect is shown for square, 4:1 rectangular and hexagon shaped paddocks (Fig 1).

The greater cost of the triangular design as both an isolated paddock or as a composite scheme would initially suggest that it was an inefficient design. But it is to be noted that six adjacent equilateral triangular paddocks form a hexagon group and are the basis of the South African 'wagon wheel' paddock system where the central section is separated off for a water-bore or stock handling facilities and which lead to easy movement of stock between paddocks.

\section{Access Lanes}

Two patterns of access lanes were considered for each of the square and hexagon pattern paddock subdivision: either branching lateral lanes, or ring lanes with a few

Figure 2 Effect of access lane configuration on total fencing costs.

BaNOH

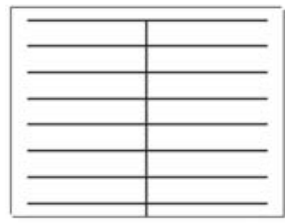

$25 \%$

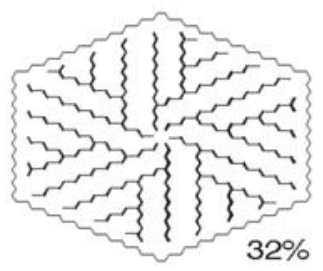

FNG

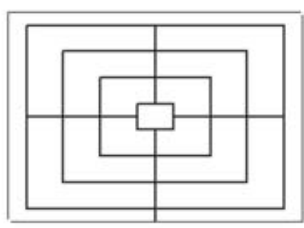

$30 \%$

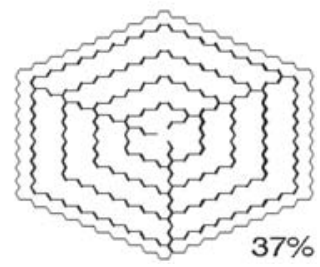

connecting cross lanes (Fig. 2).

The construction of access lanes is equivalent to double fencing of a number of lines. The simulation assumed that all paddocks were directly accessible from a lane, with each gate assembly to at least two alternative paddocks (3 or 4 at corners or end of lanes - Fig. 3).

For a square paddock subdivision the provision of the basic lane patterns adds $25 \%$ to the total needed fence lengths. However, since there is a need to provide cross access between the different ring lanes the total for that pattern was $30 \%$ greater than for a simple square subdivision.

For the hexagon paddock subdivision the efficiency of the reduced fencing length for a single or composite design is lost when lanes are included because of the greater length of zig-zag fence lines required. Counteracting the cost of provision of access lanes, is that each paddock requires only a single gate system, rather than others that would be needed to get access across to further paddocks in the absence of lanes.

The lane patterns were assessed from two perspectives of stock movement: either the mean distance (hence time and wages) in moving stock to the central facilities like yards or woolshed, or the mean distance between any pair of paddocks.

For a square paddock subdivision the mean distance from paddocks to the central farm facilities is identical for both lane patterns when those facilities are in the centre of the farm. The mean distance is marginally greater for the branching lane pattern if the farm facilities were at one edge of the farm.

For the hexagon paddock subdivision the mean distance from paddocks to the central farm facilities was $43 \%$ greater for the ring pattern with only three lateral lanes, than for the branching design. It would be less with six lateral lanes.

A random sample of the travel distance between pairs of paddocks was determined $(\mathrm{n}=280)$. For a square paddock subdivision the travel distance between any two paddocks was identical in about two thirds of the cases with either lane pattern. However when all possible transfers are considered then the mean travel distance is $9 \%$ less for the ring lane pattern than for the rectangular pattern. Thus it would be the desirable lane pattern to aim for. That was not influenced by the location of the central facilities. For the hexagon paddock subdivision the mean distance from paddocks to the central farm facilities was $9 \%$ greater for the ring pattern with only three lateral lanes, than for the branching design.

However, random movement of stock between paddocks is probably a worst case scenario. In practice one would tend to move through a series of adjacent paddocks in some rotational sequence. Thus there is probably little difference in which pattern of access lanes 
was used, though with a branching design all movements tend to be through the central facilities.

There is probably a difference in views on the needed width of access lanes. A width as narrow as $50 \mathrm{~m}$ is probably adequate for access considerations only, but prone to overgrazing and trampling of lane ways, whereas with a width of 100-200 m some of the lanes could be considered as paddocks in their own right.

\section{Strainer and Gate Systems}

A good part of the cost of subdivision fencing is in the gate-way and strainer post /stays of fence-end, fencecorner assemblies, or access from lane-ways. The strainerposts /stay systems can be of three types - those that can be temporary during construction of successive stages

Figure 3 Paddock access from lanes - possible post and gate requirements (end posts $(x)$ + gate posts (o)).

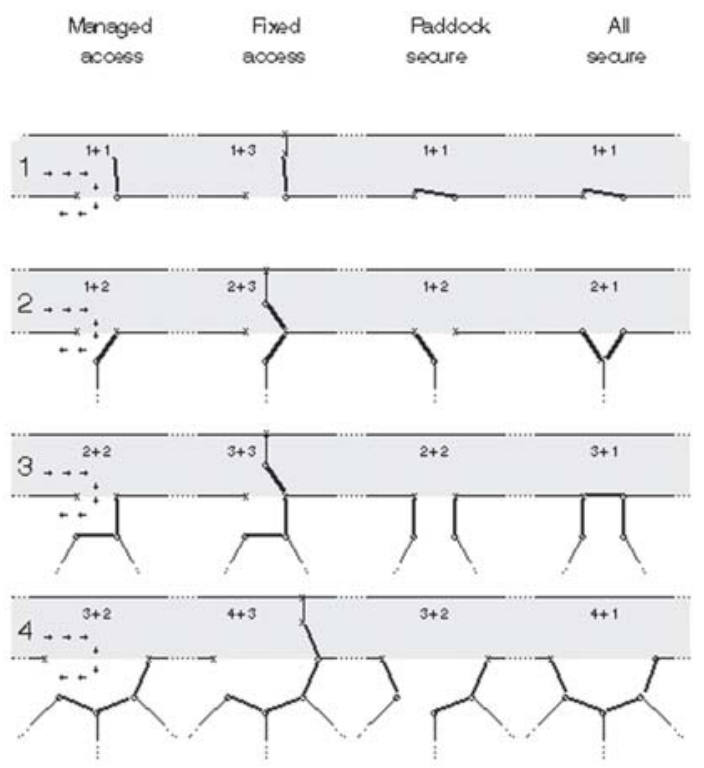

of a fence, those that are fence-ends or corners, and those of needed strength to support a swinging gate.

Possible strainer post and gate requirements for access to a different number of paddocks from a lane system are indicated (Fig. 3). (For ease in diagrammatic representation the lanes are shown narrower and gates wider than they would be in reality and with desired access to the left most paddock in each set).

The possibilities are given for four scenarios - a managed access where stock can be diverted into the appropriate open gate (having a dog!); where there are choke points or gates that can be set within the lane system so that access is fixed and predetermined; where the stock can be secured within the particular paddocks once there, even though that may result in adjacent paddocks being left open; or where all paddocks are fully secure following access. There is an increasing need for gates and gate strainer-posts for the successive scenarios.

The provision of fixed access from the lane-ways requires greatly increased strainer assemblies and gates. In effect they are almost equivalent to another subdivision and partly support the view that lanes can be sufficiently wide that they can be used as paddocks. The provision of fixed access by lane-way gates is only shown for one scenario, but also applicable to the two alternatives for paddock security with the increase in strainer and gate requirements. Access to the two or three paddock scenarios is independent of direction from which stock come in the lane. However, they differ for the one and four paddock scenarios, requiring additional fencing or longer gates.

\section{Conclusion}

The paper has demonstrated that there are some geometric considerations in the shape of paddocks and provision of access lanes, in the total fencing costs and ease of management in subdivision fencing. 